\title{
Effect of Dispersants on the Sorption Isotherm of Nonionic Dyes on Polymers from Water
}

\section{Takao Shibusawa}

Department of Biological and Chemical Engineering, Faculty of Technology, Gunma University, 1-5-1, Tenjin-cho, Kiryu-shi, 376 Japan

\begin{abstract}
Disperse dyes dissolved in aqueous dye bath bind to dispersants; the bound dye loses the affinity for polymer substrates. Addition of a constant concentration of dispersants in the dye bath varies the shape of the sorption isotherm of disperse dyes on polymers due to the dye binding by dispersants.

Computer simulation predicted that the shape of the isotherm will vary from convex to upward to linear and then to concave to upward as the concentration of the dispersant is increased. The prediction was confirmed experimentally using $\beta$-cyclodextrin as a model compound for dispersants.

Linear sorption isotherms of purified disperse dyes on polymers reported by earlier investigators were interpreted in terms of the presence of a constant concentration of dispersants in aqueous dye bath.
\end{abstract}

\section{Introduction}

Sorption isotherms of purified dyes on polymers provide fundamental data to clarify the mechanism of dyeing [1]. Earlier investigators reported that sorption isotherms of purified azo disperse dyes on hydrophobic substrates from water are linear up to the saturation in water; the isotherms meet the origin $[2-6]$. The mechanism of the dye sorption was assigned to simple partitioning of dye between polymer and water accordingly. In these cases, a constant concentration of a dispersant was often added in dye bath to facilitate the dissolution of dye in the bath $(2-6)$.

Recently, however, we have found that the sorption isotherms of purified disperse dyes on various polymers from water in the absence of dispersants are usually curved, convex upward, particularly in the range of low dye concentration $C_{S}$ in water $[7-10]$. The isotherms were well described in terms of the dual-mode sorption model expressed by Eq.1 (7-10).

$$
\mathrm{C}_{\mathrm{f}}=\mathrm{C}_{\mathrm{P}}+\mathrm{C}_{\mathrm{L}}=\mathrm{K}_{\mathrm{P}} \mathrm{C}_{\mathrm{S}}+\frac{\mathrm{K}_{\mathrm{L}} \mathrm{SC}_{\mathrm{S}}}{1+\mathrm{K}_{\mathrm{L}} \mathrm{C}_{\mathrm{S}}}
$$

where $C_{p}$ and $C_{2}$ are the equilibrium concentration of dye on polymer sorbed by Nernst type partitioning and Langmuir sorption, respectively; $S$ is the saturation value for Langmuir sorption and $\mathrm{K}_{\mathrm{L}}$ is the Langmuir constant.
The discrepancy of our results from those of earlier investigators may come from the experimental conditions, whether dispersants are added in the dye bath or not The disperse dye dissolved in dye bath binds to dispersants, (polyelectrolytes or surface active agents) [12-13], and loses its affinity to hydrophobic polymers. The binding of the dye by dispersants may change the slope $[2,4]$ and the shape of the sorption isotherms of dyes. Dispersants are sorbed by polymers from dye bath. Therefore, it is difficult to maintain the actual concentra. tion of dispersant in the bath as a constant value during the measurements of the isotherm. We found that $\beta$-cyclodextrin $(\beta C D)$ forms $1: 1$ complex with some nonionic azo dyes in water (14). The dyes are included in the hydrophobic cavity of $\beta C D$ and lose the affinity on polymers as like the dye bound by dispersants. $\beta C D$ is not absorbed by polymer, therefore we can clarify the effect of addition of dispersants on the sorption isotherm of disperse dyes using $\beta C D$ instead of dispersants.

In the present paper, the change in the available dye concentration for dyeing in dye bath by addition of a constant concentration of dispersant is calculated and then the effect of addition of $\beta \mathrm{CD}$ on the shape of the isotherm of a nonionic azo dye on Nylon-6 is experimentally clarified. 


\section{Experimental}

Dye and polymer: the structure of purified azo dye used is shown below.

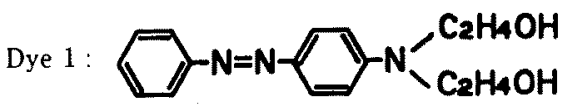

The dye and Nylon-6 film are identical with those used in the previous work [ 7$], \beta$-cyclodextrin (purity , 97\%) was purchased from Wako Pure Chemical Indus. tries Ltd., It was dried in Vacuo at $80^{\circ} \mathrm{C}$ for $25 \mathrm{~h}$ and then used without further purifications.

Sorption isotherms were measured by means of similar manner as used in previous works $[7-9]$. The calculations were made on a NEC-9801 RA-21 personal computer equipped with a numerical coprocessor ( 80387 ).

\section{Results and Discussion}

When a nonionic dye dissolved monomolecularly in water binds to a polyelectrolyte (dispersant) as expressed by Eq. 2, the binding constant $\mathrm{K}_{1}$ is expressed by Eq. 3.

$$
\begin{aligned}
& \text { Dye }+\beta \mathrm{CD} \stackrel{\mathrm{K}_{1}}{=} \text { bound Dye } \\
& (\mathrm{a}-\mathrm{x})(\mathrm{nP}-\mathrm{x}) \quad(\mathrm{x}) \\
& \mathrm{K}_{1}=\frac{\mathrm{x}}{(\mathrm{a}-\mathrm{x})(\mathrm{nP}-\mathrm{x})}
\end{aligned}
$$

where $a$ and $P$ are initial concentration of dye and polyelectrolyte (constituent unit basis), respectively, $\mathrm{n}$ is the number of binding site in constituent unit of polymer, and $\mathrm{x}$ is the concentration of bound dye.

The dye bound to the polyelectrolyte loses the affinity for the polymer, therefore, the concentration of dye available for dyeing is expressed by Eq. 4 .

$a-x=a-\frac{1}{2}\left(\left(a+n P+\frac{1}{K_{1}}\right)-\left|\left(a+n P+\frac{1}{K_{1}}\right)^{2}-4 a n P\right|^{1 / 2}\right)$

When the dye forms $1: 1$ complex with a surfactant, the available dye concentration in dye bath is expressed by $\mathrm{Eq} .5$, where $\mathrm{b}$ is the initial concentration of the surfactant and $K_{2}$ is the formation constant of the complex.

$a-x=a-\frac{1}{2}\left[\left(a+b+\frac{1}{K_{2}}\right)-\left\{\left(a+b+\frac{1}{K_{2}}\right)^{2}-4 a b\right\}^{1 / 2}\right]$

Fig. 1 shows relations between $a$ and $(a-x)$ in the presence of a constant concentration of dispersant, such as $\beta$-naphthalene-sulfonic acid-formaldehyde condensates. The curves were drawn by Eq.4 using $n=1$, molecular

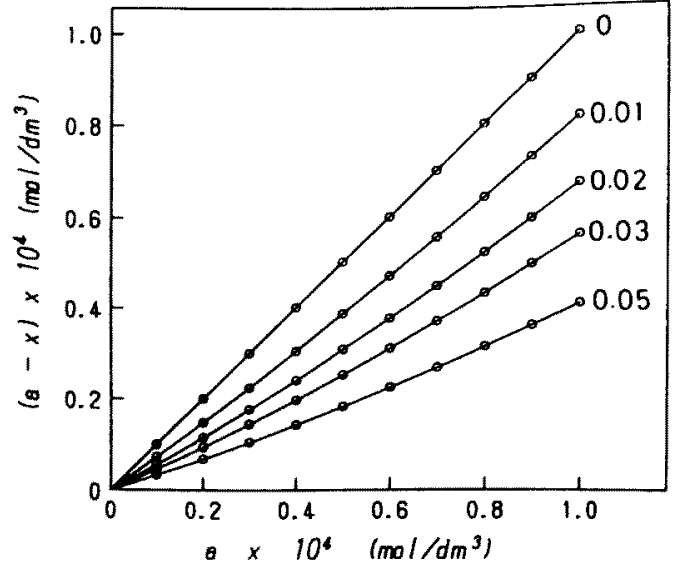

Fig. 1 Relation between total concentration of nonionic dye (a) and that available for dyeing in a dye bath $(a-x)$. Each line was calculated by $\mathrm{Eq} .4$. The numbers near the end of each line denote the concentration of dispersant in $\mathrm{g} / \mathrm{dm}^{3}$.

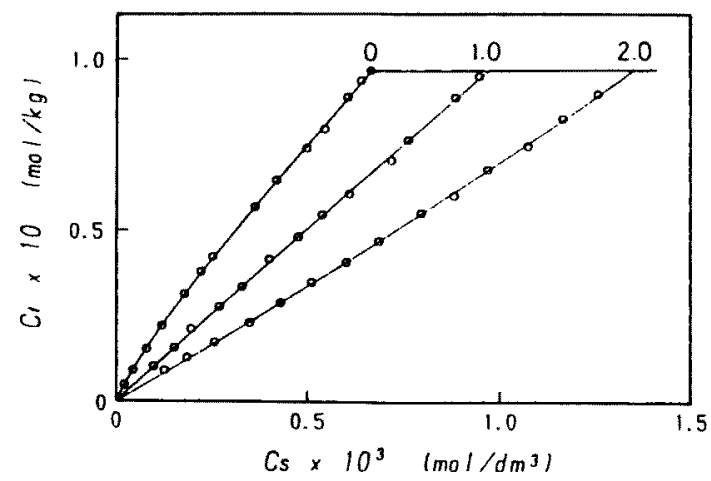

Fig. 2 Change in the sorption isotherm of Dye 1 on Nylon-6 film by addition of $\beta \mathrm{CD}$ at $60^{\circ} \mathrm{C} . \mathrm{O}$, ex. perimental point determined from dyeing in aqueous solution of dye, experimental point determined from dyeing in aqueous dispersion of dye. The numbers near the saturation points denote the concentration of $\beta C D$ in $\mathrm{g} / \mathrm{dm}^{3}$.

weight of the dispersant per constituent unit $=242.2$ and $K_{1}=10000\left(\mathrm{dm}^{3} /\right.$ base mol $)[12]$. Fig.1 indicates that if a constant partition of dye between water and substrate is valid as reported by earlier investigators [2-6], the sorption isotherm measured in the presence of a constant concentration of dispersant will be concave to upward. The curvature of the isotherm increases with increasing dispersant concentration. On the other hand, when the isotherm of a dye on a polymer is convex to upward in 
the absence of dispersant, the shape of the isotherm will be varied from the curved to linear and then to concave to upward by addition of increasing amount of dispersant.

Fig.2 shows the change in the sorption isotherm of Dye 1 by addition of constant concentration of $\beta C D$ in dye bath at $60 \mathrm{C}$. The isotherm measured in the absence of $\beta C D$ was curved, convex to upward in the range of lower $C_{S}$. A curve passing through the experimental points was drawn by Eq. 1 using $\mathrm{K}_{\mathrm{P}}=126\left(\mathrm{dm}^{3} / \mathrm{kg}\right), \mathrm{K}_{\mathrm{L}}$ $=7380\left(\mathrm{dm}^{3} / \mathrm{mol}\right)$ and $S=0.0151(\mathrm{~mol} / \mathrm{kg}) ;$ the sorp tion parameters were obtained by means of nonlinear least squares fit of experimental points to Eq. 1. The experimental points fitted well to the curve calculated.

The isotherm measured in the presence of $1.0 \mathrm{~g} / \mathrm{dm}^{3}$ of $\beta C D$ seems to be linear. A curve passing through the ex. perimental points was drawn by a polynomial in a form of $C_{f}=A_{0}+A_{1} C_{S}+A_{2} C_{S}{ }^{2}$, the constant $A_{0}$, and the coefficients $A_{1}$ and $A_{2}$ were obtained by means of the least squares fit of experimental points to the polynomial.

The isotherm measured in the presence of $2.0 \mathrm{~g} / \mathrm{dm}^{3}$ of $\beta C D$ was obviously curved, concave to upward as predicted from Fig.1 The curve passing through the experimental points was also drawn by the polynomial of degree two which was obtained as described above.

Fig. 2 clearly demonstrates that the sorption isotherms of a nonionic dye can be varied from curved (convex to upward) to almost linear by addition of a suitable con. centration of dispersants. This is the reason why earlier investigators observed linear isotherms of purified azo disperse dyes on polymers.

\section{References}

1. C. H. Giles, T. H. MacEvan. S. N. Nakhwa, and S. N. Smith, J. Chem. Soc., 3973-3993 (1960).

2. C. L. Bird and F. Manchester, Disc. Farady. Soc., 16, 85 (1954).

3. C. L. Bird and F. Manchester, J. Soc. Dyers Colour., 71, 604 (1955)

4. C. L. Bird, H. K. Partovi, and G. Tabbron, J. Soc. Dyers Colour.. 75, 600 (1959).

5. A. Johnson, R. H. Peters and, A. S. Ramadan, J. Soc. Dyers Colour., 80, 129 (1964).

6. P. Savarino, E. Barni, G. Viscardi, R. Carpignano, and G. Di. Modica, J. Soc. Dyers Colour., 104, 125 (1988).

7. T. Shibusawa and Y. Chigira, J. Polym. Sci., Part B, Polym. Phys., 30. 563 (1992)

8. T. Shibusawa and N. Arai, H. Ueno and T. Nakamura, Sen'i Gakkaishi, 48, 641 (1992).

9. T. Shibusawa and T. Nakamura, Sen'i Gakkaishi, 49. 576 (1993).

10. T. Shibusawa and T. Nakamura, Text. Res. J., in press.

11. T. Shibusawa and Y. Hirose, Sen'i Gakkaishi, 29. $T-1$ (1972).

12. T. Shibusawa and F. Igarashi, Nippon Kagakukaishi, 1974, 1544 (1974).

13. T. Shibusawa Sen'i Gakkaishi, 43, 614 (1987).

14. T. Shibusawa, T. Hamayose, and N. Sasaki, Nippon Kagakukaishi, 1975, 2171 (1975). 